Pacific Journal of Mathematics

AXIOMATIC CONVEXITY THEORY AND RELATIONSHIP
BETWEEN THE CARATHÉODORY, HELLY, AND RADON
NUMBERS

David Clifford Kay and Eugene W. Womble 


\section{AXIOMATIC CONVEXITY THEORY AND RELATIONSHIPS BETWEEN THE CARATHÉODORY, HELLY, AND RADON NUMBERS}

\section{David C. Kay and Eugene W. Womble}

An axiomatic setting for the theory of convexity is provided by taking an arbitrary set $X$ and constructing a family $\mathscr{C}$ of subsets of $X$ which is closed under intersections. The pair consisting of any ordered vector space and its family of convex subsets thus become the prototype for all such pairs $(X, \mathscr{C})$. In this connection, Levi proved that a Radon number $r$ for $\mathscr{C}$ implies a Helly number $h \leqq r-1$; it is shown in this paper that exactly one additional relationship among the Carathéodory, Helly, and Radon numbers is true, namely, that if $\mathscr{C}$ has Caratheodory number $c$ and Helly number $h$ then $\mathscr{C}$ has Radon number $r \leqq c h+1$. Further, characterizations of (finite) Caratheodory, Helly, and Radon numbers are obtained in terms of separation properties, from which emerges a new proof of Levi's theorem, and finally, axiomatic foundations for convexity in euclidean space are discussed, resulting in a theorem of the type proved by Dvoretzky.

1. Preliminary definitions. A family of subsets of a space $X$ which is closed under intersection yields a weak type of closure, or hull, operator on the power set of $X$, producing concepts which may be readily applied to convexity and topology alike. Our main interest is, however, convexity theory and the abstraction of certain classical concepts from that area. (See in this regard the papers by Danzer, Grünbaum and Klee [1], Hammer [5, 6, 7], Eckhoff [3], Ellis [4], Koenen [8], and Levi [9].) We shall, therefore, introduce the following terminology: A family $\mathscr{C}$ of subsets of a set $X$ is termed a convexity structure for $X$, with the pair $(X, \mathscr{C})$ being called a convexity space, whenever the following two conditions hold:

(a) $\varnothing$ and $X$ belong to $\mathscr{C}$;

(b) $\cap \mathscr{F} \in \mathscr{C}$ for each subfamily $\mathscr{F} \subset \mathscr{C}$.

$\mathscr{C}$ is designated $T_{1}$ iff the further condition

(c) $\{x\} \in \mathscr{C}$ for each $x \in X$

holds, and a subfamily $\mathscr{B}$ of $\mathscr{C}$ is called a basis of $\mathscr{C}$ iff each member of $\mathscr{C}$ is obtainable as an intersection of members of $\mathscr{B}$.

The hull operator generated by a convexity structure $\mathscr{C}$, defined in the usual manner by the relation

$$
\mathscr{C}(S)=\bigcap\{C \in \mathscr{C}: C \supset S\}, \quad S \subset X,
$$


enjoys certain properties identical to those of the closure operator in topology, among which are: (i) $S \subset \mathscr{C}(S)$ for each $S \subset X$; (ii) if $S_{1} \subset S_{2}$ the $\mathscr{C}\left(S_{1}\right) \subset \mathscr{C}\left(S_{2}\right)$; (iii) $\mathscr{C}(\mathscr{C}(S))=\mathscr{C}(S)$; and, (iv) $S \in \mathscr{C}$ iff $\mathscr{C}(S)=S$. The set $\mathscr{C}(S)$ will be termed the $\mathscr{C}$-hull of $S$, and a set will be called $\mathscr{C}$-convex iff $\mathscr{C}(S)=S$. If $S$ is finite and consists of the points $x_{1}, \cdots, x_{k}$ we shall write simply $\mathscr{C}\left(x_{1}, \cdots, x_{k}\right)$ for its $\mathscr{C}$-hull.

An important concept in ordinary convexity theory is that of the "cone" or "join" of a point over a set. We may extend this concept to our general setting by defining the $\mathscr{C}$-join of $x$ and $S$ to be the set

$$
x_{\mathscr{C}} S \equiv \bigcup_{s \in S} \mathscr{C}(x, s)
$$

A useful condition involving this concept is the following (for $x \in X$ and $S \subset X)$ :
(d) $\mathscr{C}(x \cup S) \subset x_{\mathscr{E}} \mathscr{C}(S)$.

Since the reverse inclusion can be easily proved, (d) is equivalent to the condition

$\left(\mathrm{d}^{\prime}\right) \quad \mathscr{C}(x \cup S)=x_{\mathscr{C}} \mathscr{C}(S)$.

It is interesting that (d) is also equivalent to assuming that the $\mathscr{C}$-join and $\mathscr{C}$-hull operators commute at $x \in X$, that is, for each $S \subset X$,

$$
\left(\mathrm{d}^{\prime \prime}\right) \mathscr{C}\left(x_{\mathscr{E}} S\right)=x_{\mathscr{B}} \mathscr{C}(S)
$$

This may be seen by simply verifying the relation $\mathscr{C}(x \cup S)=\mathscr{C}\left(x_{\mathscr{\delta}} S\right)$. A convexity structure satisfying either of the equivalent conditions (d), $\left(\mathrm{d}^{\prime}\right)$, or $\left(\mathrm{d}^{\prime \prime}\right)$ will be called join-hull commutative at $x$, and if $\mathscr{C}$ is join-hull commutative for each $x \in X$ it will be said to be joinhull commutative. Further, we say that $\mathscr{C}$ is finitely join-hull commutative if $(\mathrm{d}),\left(\mathrm{d}^{\prime}\right)$, or $\left(\mathrm{d}^{\prime \prime}\right)$ holds for each $x \in X$ and for each finite subset $S \subset X$. (Condition (d) was introduced for finite subsets by Ellis [4].)

The next property is the direct analogue of the classical Carathéodory theorem on convex hulls in a vector space over an ordered field, and will reveal a relationship between join-hull commutativity and finite join-hull commutativity:

(e) $\mathscr{C}(S)=\bigcup\{\mathscr{C}(T): T \subset S,|T|<\infty\}$ for each $S \subset X$.

After Hammer [6], a convexity structure $\mathscr{C}$ having property (e) will be termed domain finite.

The following two theorems will illustrate the application of these properties.

THEOREM 1. If $\mathscr{C}$ is a convexity structure for $X$ which is domain finite, then finite join-hull commutativity implies join-hull com- 
mutativity.

Proof. It suffices to show that for $x \in X$ and $S \subset X, \mathscr{C}(x \cup S) \subset$ $x \in \mathscr{C}(S)$. Let $y \in \mathscr{C}(x \cup S)$; then there exists a finite set $T \subset S$ such that $y \in \mathscr{C}(x \cup T)$ and

$$
\mathscr{C}(x \cup T) \subset x_{\mathscr{E}} \mathscr{C}(T) \subset x_{\mathscr{E}} \mathscr{C}(S) .
$$

Hence, $y \in x_{\odot} \mathscr{C}(S)$.

THEOREM 2. If $\mathscr{C}$ is a convexity structure for $X$ which is joinhull commutative and domain finite, then a set $C \subset X$ is $\mathscr{C}$-convex iff $\mathscr{C}(x, y) \subset C$ for each $x \in C, y \in C$.

Proof. Suppose $C$ is $\mathscr{C}$-convex. Then if $x \in C$ and $y \in C$, $\mathscr{C}(x, y) \subset \mathscr{C}(C)=C$. Conversely, suppose for each $x \in C$ and $y \in C$, $\mathscr{C}(x, y) \subset C$; we observe that the hypothesis implies by join-hull commutativity that for any finite set $T \subset C, \mathscr{C}^{2}(T) \subset C$. It follows immediately that $\mathscr{C}(C)=C$, for, by domain finiteness,

$$
\mathscr{C}(C)=\bigcup\{\mathscr{C}(T): T \subset C,|T|<\infty\} \subset C .
$$

Finally, a convexity structure $\mathscr{C}$ is said to have Caratheodory number $c$ iff $c$ is the smallest positive integer for which it is true that the $\mathscr{C}$-hull of any set $S \subset X$ is the union of the $\mathscr{C}$-hulls of those subsets of $S$ of cardinality $\leqq c$. Further, a convexity structure has Helly number $h$ and Radon number $r$ iff $h$ and $r$ are the smallest positive integers for which it is true that, respectively, a finite subfamily $\mathscr{F}$ of sets in $\mathscr{C}$ has nonempty intersection provided each $h$ members of $\mathscr{F}$ has nonempty intersection, and any set $S$ with $|S| \geqq r$ has a Radon partition, that is, may be partitioned into two nonempty subsets $\left(S_{1}, S_{2}\right)$ such that $\mathscr{C}\left(S_{1}\right) \cap \mathscr{C}\left(S_{2}\right) \neq \varnothing$.

These definitions imply that in general $c \geqq 1, h \geqq 1$, and $r \geqq 2$, and that for any $T_{1}$ convexity space having at least 3 points, $c \geqq 1$, $h \geqq 2$, and $r \geqq 3$. The least value for $c$ in either case is attained by taking $\mathscr{C}$ to be the largest possible convexity structure for $X$ (consisting of the power set of $X$ ), and the least values for $h$ and $r$ are obtained when $\mathscr{C}$ is the smallest possible $\left[T_{1}\right]$ convexity structure (consisting of $\varnothing$, [the singleton subsets of $X$ ], and $X$ ). If $\mathscr{C}$ is the family of convex sets in euclidean space $E^{d}$ of dimension $d$ the classical theorems of Carathéodory, Helly, and Radon imply that in this case $\mathscr{C}$ has $c=h=d+1$ and $r=d+2$. It is easy to construct examples to show that convexity structures can have a variety of possible Carathéodory, Helly, and Radon numbers, but in general there will be certain restrictions. 
2. Interrelationships between the numbers $c, h$, and $r$. Levi's theorem [9] shows that in any convexity space $(X, \mathscr{C})$ if $\mathscr{C}$ has Radon number $r$ then $\mathscr{C}$ has Helly number $h \leqq r-1$. To show that no other possible relationships between the numbers $c, h$, and $r$ exist (taken singly) we cite the following examples (also discussed in part using different definitions by $M$. Breen in a related unpublished paper communicated to the authors by W. R. Hare and J. W. Kenelly): Take $X=E^{2}$ and consider Hammer's example of the convexity structure generated by $X$ and sets of the form $C=H_{1} \cup H_{2} \cup\left(L_{1} \sim L_{2}\right)$, where $L_{1}$ and $L_{2}$ are any two perpendicular lines and $H_{1}$ and $H_{2}$ are open half planes determined by them. As proved in [5], this convexity structure has Carathéodory number 7 but no finite Helly or Radon number. The example consisting of $X=E^{d}$ and all closed convex sets in $X$ provides a convexity structure which has Helly number $d+1$, Radon number $d+2$, but no finite Carathéodory number (since no point on the boundary of an open convex set $S$ is contained in the closed, convex hull-and thus $\mathscr{C}$-hull-of any finite subset of $S$ ).

The above two examples show that among the Caratheodory, Helly, and Radon numbers $c, h$, and $r$, the existence of $c$ does not imply that of either $h$ or $r$, and neither the existence of $h$ nor $r$ implies that of $c$. It remains to show that the existence of $h$ does not imply that of $r$. To that end, consider the following example.

EXAmple 1. In the sequence space $X=E^{\infty}=\left\{\left(x_{1}, \cdots, x_{i}, \cdots\right)\right.$ : $\left.x_{i} \in R\right\} \quad(R=$ reals $)$, let $\mathscr{C}$ consist of $\varnothing, X$, and the collection of all closed and bounded rectangular hypersolids with faces orthogonal to the coordinate axes, explicitly defined as $C \equiv \bigcap_{i \in N} C_{i} \quad(N=$ positive integers), where, for each $i$,

$$
C_{i}=\left\{x: a_{i} \leqq x_{i} \leqq b_{i}\right\},
$$$$
a_{i} \leqq b_{i},
$$

$x_{i}$ denoting the $i^{\text {th }}$ coordinate of $x$. (This is Eckhoff's product $\Pi_{i=1}^{\infty}\left(X_{i}, \mathscr{C}_{i}\right)$ with $X_{i}=E^{1}$ and $\mathscr{C}_{i}=$ family of closed intervals; see [3] and a related paper by Reay [10].)

It is clear that if $(X, \mathscr{C})$ is the convexity space defined in Example 1 and $S \subset X$,

$$
\mathscr{C}(S)=\left\{x: a_{i} \leqq x_{i} \leqq b_{i}, i \in N\right\},
$$

where

$$
a_{i}=\inf _{x \in S} x_{i}, \quad b_{i}=\sup _{x \in S} x_{i} .
$$

From reasoning of a similar nature it follows that $\mathscr{C}$ has Helly number 2. But we show that $\mathscr{C}$ has no (finite) Radon number. Let 
$k \in N, k \geqq 2$, and with $n=\sum_{j=1}^{k-1}\left(\begin{array}{c}k \\ j\end{array}\right)=2^{k}-2$, construct the $k \times n$ matrix $M_{k}$ of zeroes and ones as follows: The first $\left(\begin{array}{l}k \\ l\end{array}\right)$ columns define the characteristic functions of all one-element subsets of $\{1, \cdots, k\}$, the next $\left(\begin{array}{l}k \\ 2\end{array}\right)$ columns define the characteristic functions of all twoelement subsets, and in general, the $\left(\begin{array}{l}k \\ l\end{array}\right)$ columns $1+\sum_{j=1}^{l-1}\left(\begin{array}{l}k \\ j\end{array}\right)$ through $\sum_{j=1}^{l}\left(\begin{array}{l}k \\ j\end{array}\right)$ define the characteristic functions of all $l$-element subsets of $\{1, \cdots, k\}, 1 \leqq l \leqq k-1$. Note that for $k=5$ (in which case $n=30$ ), this process yields the $5 \times 30$ matrix

$$
M_{5}=\left[\begin{array}{llll}
10000 & 1111000000 & 1111110000 & 11110 \\
01000 & 1000111000 & 1110001110 & 11101 \\
00100 & 0100100110 & 1001101101 & 11011 \\
00010 & 0010010101 & 0101011011 & 10111 \\
00001 & 0001001011 & 0010110111 & 01111
\end{array}\right] .
$$

Now let $S$ be the $k$-element subset of $X$ defined by taking those points whose first $n$ coordinates are given by the rows of $M_{k}$ and whose remaining coordinates are each zero. If $\left(S_{1}, S_{2}\right)$ is any nontrivial partitioning of $S$ then $1 \leqq\left|S_{1}\right| \leqq k-1$ and there is an integer $i$ such that the $i^{\text {th }}$ coordinate of each member of $S_{1}$ is 1 and the $i^{\text {th }}$ coordinate of each member of $S_{2}$ is 0 . Thus, the $i^{\text {th }}$ coordinate of each member of $\mathscr{C}\left(S_{1}\right)$ is 1 , and the $i$-th coordinate of each member of $\mathscr{C}\left(S_{2}\right)$ is 0 , from which it follows that $\mathscr{C}\left(S_{1}\right) \cap \mathscr{C}\left(S_{2}\right)=\varnothing$. Therefore, no $k$-element subset of $X$ has a Radon partition, and since $k$ was arbitary, $\mathscr{C}$ has no radon number.

The preceding examples show that Levi's theorem is the only one possible if we assume the finiteness of exactly one of the numbers $c, h$, or $r$; however a possible implication arises by considering pairs of numbers $c, h$, or, $r$ and this is answered conclusively by the following theorem.

THEOREM 3. If $\mathscr{C}$ is a convexity structure for $X$ which has Caratheodory number $c$ and Helly number $h$, then $\mathscr{C}$ possesses a Radon number $r \leqq c h+1$.

Proof. Let $S$ be a $(c h+1)$-element subset of $X$, and define $\mathscr{F}$ to be the subsets of $S$ having at least $c h+1-c$ clements. By a simple counting argument it follows that each $h$ members of the family $\mathscr{G}=\{\mathscr{C}(F): F \in \mathscr{F}\} \subset \mathscr{C}$ have nonempty intersection: Let $G_{i}=\mathscr{C}\left(F_{i}\right)$ for each $F_{i} \in \mathscr{F}, i=1, \cdots, h$, and consider $\left|\bigcup_{i}\left(S \sim G_{i}\right)\right| \leqq$ $\bigcup_{i}\left|S \sim G_{i}\right| \leqq \bigcup_{i}\left|S \sim F_{i}\right| \leqq h[(c h+1)-(c h+1-c)] \leqq c h<|S|$. 
Since $\mathscr{C}$ has Helly number $h$ there is a point $x$ belonging to $\cap \mathscr{G}$, and because $S \in \mathscr{F}$ and $\mathscr{C}(S) \in \mathscr{G}$ we have $x \in \mathscr{C}(S)$. But $\mathscr{C}$ has Carathéodory number $c$ and therefore we can find a subset $T$ of $S$ of cardinality $c$ or less such that $x \in \mathscr{C}(T)$. Since $S \sim T$ has cardinality at least $c h+1-c$ then $\mathscr{C}(S \sim T) \in \mathscr{C}$ and $x \in \mathscr{C}(S \sim T)$. Hence, the pair $(T, S \sim T)$ is a Radon partition of $S$, proving that $\mathscr{C}$ has Radon number $r \leqq c h+1$.

CoRollary 1. In a convexity space having finite Carathéodory number $c$, the existence of a Helly number $h$ and a Radon number $r$ are equivalent, and the corresponding numbers satisfy the inequality

$$
h+1 \leqq r \leqq c h+1 \text {. }
$$

The following example due to Eckhoff [3] sheds further light on the general behavior of the Carathéodory, Helly, and Radon numbers.

ExAmple 2. With $X=E^{d}$ and for a given integer $k>0$ let $\mathscr{C}$ consist of all the convex subsets of $X$ and all finite subsets $S \subset X$ such that $|S| \leqq k$.

Since $\mathscr{C}$ contains the usual convex subsets of $E^{d}$ it is clear that $\mathscr{C}$ has Carathéodory number $c=d+1$. Eckhoff proves that if $2 \leqq d \leqq k+1$, then $\mathscr{C}$ has Radon number $2 k+2$, and by Levi's theorem $\mathscr{C}$ has finite Helly number $h$. By our Theorem 3 ,

$$
2 k+2=r \leqq h(d+1)+1 \text {. }
$$

Thus, by allowing $k \rightarrow \infty$ we have a class of convexity structures in which the Carathéodory number is a constant (as small as 3) while both the Helly and Radon numbers take on arbitrarily large values.

3. A characterization of the numbers $c, h$, and $r$ by separation properties. Since separation theorems bear prominently on problems in convexity, it is of interest to know how they may be related to the Carathéodory, Radon, and Helly properties in a more general setting. If two members $H_{1}$ and $H_{2}$ of a convexity structure $\mathscr{C}$ for $X$ partition $X$ they are called complementary $\mathscr{C}$-half-spaces. If $S_{1}, S_{2}$ are respectively contained by a complementary pair $H_{1}, H_{2}$ of $\mathscr{C}$-half-spaces, then $S_{1}$ and $S_{2}$ are said to be $\mathscr{C}$-separated.

The existence of $\mathscr{C}$-half-spaces and the possibility of separating disjoint members of $\mathscr{b}$ in general is a problem discussed by Ellis in [4], where he introduces a property which, together with join-hull commutativity and domain finiteness, will guarantee such separation. For our purposes, let us say that a convexity structure $\mathscr{C}$ has the separation property if it satisfies the axiom 
(f) Each two disjoint members of $\mathscr{C}$ may be $\mathscr{C}$-separated.

Following Hammer, a closely related idea is the following: $H^{\prime}$ is called a $\mathscr{C}$-semispace iff it is a member of $\mathscr{C}$ which is maximal with respect to being disjoint from some other member of $\mathscr{C}$. An application of Zorn's lemma shows that for each two disjoint members $C_{1}$ and $\mathrm{C}_{2}$ of $\mathscr{C}$ there exists a $\mathscr{C}$-semispace containing $C_{1}$ and disjoint from $C_{2}$, provided $\mathscr{C}$ is closed under unions of chains of its members (it can be proved that such is the case if $\mathscr{C}$ is domain finite). Thus, mere existence of $\mathscr{C}$-semispaces in $\mathscr{C}$ is no problem, but the complement of a $\mathscr{C}$-semispace may not be a member of $\mathscr{C}$. It turns out that an alternate way to handle the separation problem is to assume the following property, which can shown to be equivalent to (f) above in domain finite convexity structures:

$\left(f^{\prime}\right)$ The complement of each $\mathscr{C}$-semispace is $\mathscr{C}$-convex.

It is then clear that whenever (f) [or $\left(f^{\prime}\right)$ ] is assumed in a domain finite convexity structure $\mathscr{C}$, with $\mathscr{S}$ and $\mathscr{C}$ denoting the $\mathscr{C}$-semispaces and $\mathscr{C}$-half-spaces of $\mathscr{C}, \mathscr{S} \subset \mathscr{H} \subset \mathscr{C}$; moreover, if $\mathscr{C}$ is $T_{1}$ then $\mathscr{S}$ and $\mathscr{X}$ are both bases for $\mathscr{C}$, with the members of $\mathscr{H}$ being generated by those of $\mathscr{S}$.

We now proceed to the characterization theorems mentioned earlier; the first two do not require domain finiteness.

Theorem 4. In any $T_{1}$ convexity structure $\mathscr{C}$ on $X$ having the separation property, the following two conditions are equivalent:

(i) $\mathscr{C}$ has Helly number $h \leqq k$.

(ii) If $S$ is a $(k+1)$-element subset of $X, k \geqq 2$, there exists $p \in X$ such that every $\mathscr{C}$-half-space containing at least $k$ elements of $S$ also contains $p$.

Proof. (i) $\rightarrow$ (ii). If $S$ is any $(k+1)$-element subset of $X$, form the sets $S_{i}=S \sim\left\{x_{i}\right\}$, where $x_{i} \in S$, and the family $\mathscr{F}=\left\{\mathscr{C}\left(S_{i}\right)\right\} \subset \mathscr{C}$, for $i=1, \cdots, k+1$. Since each $k$ members of $\mathscr{F}$ have nonempty intersection and $\mathscr{C}$ has Helly number $h \leqq k$, there is a point $p \in \cap \mathscr{F}$. Hence if $H$ is a $\mathscr{C}$-half-space containing $k$ elements of $S, H$ contains one of the sets $\mathscr{C}\left(S_{i}\right)$ of $\mathscr{F}$, and hence $p$.

(ii) $\rightarrow$ (i). Suppose (ii) is satisfied for some $k \geqq 2$, and that (i) fails for that $k$. Then there is a smallest subfamily $\mathscr{F}$ of $\mathscr{C}$ for which it fails, with each $k$ members thereof having nonempty intersection but $\cap \mathscr{F}=\varnothing$. Hence $|\mathscr{F}| \geqq k+1$, and it follows by standard arguments and the minimal property of $\mathscr{F}$ that $|\mathscr{F}|=$ $k+1$. Let $C_{1}, \cdots, C_{k+1}$ be the members of $\mathscr{F}$, and for each $i=$ $1, \cdots, k+1$, choose $x_{i} \in \bigcap\left\{C_{j}: j \neq i\right\}$. Then $x_{i} \neq x_{j}$ for $i \neq j$, for otherwise $x_{j} \in \bigcap \mathscr{F}$. Hence let $p$ be as in (ii) with $S=\left\{x_{1}, \cdots, x_{k+1}\right\}$ and suppose $p \notin C_{l}$ for some $l$. By the separation property there is a 
$\mathscr{C}$-half-space $H$ containing $C_{l}$ but not $p$. But $C_{l}$, and therefore $H$, contains $k$ members of $S$, so by (ii) $H$ contains $p$, a contradiction.

THEOREM 5. In any $T_{1}$ convexity structure $\mathscr{C}$ on $X$ having the separation property, the following two conditions are equivalent:

(i) $\mathscr{C}$ has Radon number $r \leqq k$.

(ii) If $S$ is a k-element subset of $X, k \geqq 3$, there exists a proper subset $T$ of $S$ and $p \in X$ such that every $\mathscr{C}$-half-space which contains either $T$ or $S \sim T$ also contains $p$.

Proof. Obvious since $\mathscr{C}(T)=\bigcap\{H \in \mathscr{K}: H \supset T)$ and $\mathscr{C}(S \sim T\}=$ $\bigcap\{H \in \mathscr{L}: H \supset S \sim T\}$. Hence $\mathscr{C}(T) \cap \mathscr{C}(S \sim T)$ is nonempty iff every $\mathscr{C}$-half-space containing $T$ meets every $\mathscr{C}$-half-space containing $S \sim T$ at some point $p$.

The Carathéodory number may also be formulated in terms of separation properties, but the additional property of domain finiteness is needed. Since the proof is a routine application of the definitions it will be omitted.

THEOREM 6. Let $\mathscr{C}$ be a $T_{1}$ convexity structure for $X$ which has the separation property and is domain finite. Then the following conditions are equivalent:

(i) $\mathscr{C}$ has Carathéodory number $c \leqq k$.

(ii) If $S$ is a subset of $X$ having at least $k+1$ elements and $p \in \mathscr{C}(S)$, there is a proper subset $T$ of $S$ such that every $\mathscr{C}$-halfspace containing $T$ also contains $p$.

We now apply two of the above characterization theorems to obtain an alternate proof of Levi's theorem (in a less general setting).

THEOREM 7 (Levi). Let $\mathscr{C}$ be a $T_{1}$ convexity structure for $X$ which has the separation property. Then a Radon number $r$ for $\mathscr{C}$ implies a Helly number $h \leqq r-1$.

Proof. Let $S$ be an $r$-element subset of $X$. By property (ii) of Theorem 5 there exists $T \subset S$ and $p \in X$ such that every $\mathscr{C}$-half-space $H$ containing either $T$ or $S \sim T$ contains $p$. Let $H$ be any $\mathscr{C}$-halfspace containing $r-1$ points of $S$. Then it follows that $H$ contains either $T$ or $S \sim T$, and thus $p$, yielding property (ii) of Theorem 4 . Hence, $\mathscr{C}$ has Helly number $h \leqq r-1$.

REMARK. In view of the simple proof of Levi's theorem using 
separation properties one suspects there are additional relationships among the numbers $c, h$, and $r$ in convexity spaces satisfying the above two properties (e) and (f).

The next result makes use of certain separation properties to show that under certain conditions the existence of a Radon number is a sufficient condition for the existence of a Carathéodory number. The property needed is known to be true for $X=E^{d}$ when $d=$ $1,2,3$, with $\mathscr{C}$ the usual convexity structure (for a discussion of related versions of generalizations of Radon's theorem, see [1, p. 118]).

(g) If $S$ is a finite subset of $X$ which has a Radon partition $\left(S_{1}, S_{2}\right)$ and $p \in \mathscr{C}(S)$ but $p \notin \mathscr{C}\left(S_{1}\right) \cap \mathscr{C}\left(S_{2}\right)$, then $S$ has a Radon partition $\left(T_{1}, T_{2}\right)$ such that

$$
\bigcap\left\{H \in \mathscr{\mathscr { C }}: H \supset T_{1}, p \notin H\right\} \cap T_{2} \neq \varnothing .
$$

THEOREM 8. Let $\mathscr{C}$ be a $T_{1}$ convexity structure for $X$ having the separation property, domain finiteness, and the additional property (g) mentioned above. Then, if $\mathscr{C}$ has Radon number $r<\infty$, it has Carathéodory number $c \leqq r-1$.

Proof. Let $G$ be a subset of $X$ and $p \in \mathscr{C}(G)$; by domain finiteness there is a finite subset $S \subset G$ of minimal cardinality such that $p \in \mathscr{C}(S)$. If $|S| \geqq r$, then $S$ has a Radon partition; so by hypothesis $S$ has a Radon partition $\left(T_{1}, T_{2}\right)$ for which there exists a point $q \in \bigcap\left\{H \in \mathscr{H}: H \supset T_{1}, p \notin H\right\} \cap T_{2}$. Let $H$ be any $\mathscr{C}$-half-space containing $S \sim q$. Then $T_{1} \subset H$ (since $q \in T_{2}$ ), and if $q \notin H$, from the choice of $q$ it follows that $p \in H$; but if $q \in H$ then $S \subset H$ and again $p \in H$. Thus, $p \in \mathscr{C}(S \sim q)$, denying the minimal property of $S$. Therefore, $|S|<r$, and $\mathscr{C}$ has Carathéodory number $c \leqq r-1$.

4. An axiomatic foundation for convexity in euclidean space. It is of fundamental interest to derive the convexity structure of euclidean space from an abstract convexity structure in the case $X=E^{d}$. This can be done by assuming the axioms below [in addition to the previous conditions (a) and (b)]. Since the system is independent it can be proved that this set of conditions is both necessary and sufficient. A more difficult problem arises if we do not assume a euclidean setting, or if the axioms themselves are stated intrinsically-that is, solely in terms of the members of $\mathscr{C}$. The more general problem of deriving necessary and sufficient conditions for a convexity space $(X, \mathscr{C})$ to be a vector space over an ordered field for which the members of $\mathscr{C}$ are the convex sets might be referred to as the linearization problem for convexity.

A similarity transformation, or similitude, is any transformation 
$f: E^{d} \rightarrow E^{d}$ having the dilation ("contraction-expansion") property

$$
e(f(x), f(y))=\alpha e(x, y), \quad \alpha>0,
$$

where $e$ denotes the euclidean metric. The geometric properties of such mappings are well known, and we do not state them here. A direct similitude is one for which the matrix representing $f$ in the usual manner has positive determinant. Throughout the section, we assume that $X=E^{d}$, and the usual topology will be understood.

Axiom 1: $\mathscr{C}$ is closed under similitudes in $X$. in $X$.

Axiom 2: $\mathscr{C}$ has a member of cardinality $\geqq 2$ which is bounded

Axiom 3: For every finite set $S \subset X$, if $x \in \operatorname{cl} \mathscr{C}(S)$ then $\mathscr{C}(x \cup S) \subset x_{\mathscr{C}} \mathscr{C}(S){ }^{1}$

Axiom 4: $\mathscr{C}$ has Helly number $h \leqq d+1$.

Axiom 5: $\mathscr{C}$ is domain finite.

The first four axioms are needed to prove that the members of $\mathscr{C}$ are each convex. This constitutes a theorem of the type discovered by Dvoretzky [2], in that, the classical Helly property for $E^{d}$ is used to derive convexity. The similarity ends there, for while Dvoretzky uses the assumption that $\mathscr{C}$ is closed under affine mappings (stronger than our Axiom 1) and the compactness of its members (stronger than our Axiom 2), he does not assume the very restrictive condition that $\mathscr{C}$ is closed under arbitrary intersection. The closure of $\mathscr{C}$ under intersections coupled with closure under affine mappings is quite strong indeed; for, it is not difficult to prove that the only additional assumption needed to obtain the convexity of each member of $\mathscr{C}$ is, for example, that the $\mathscr{C}$-hull of two points be connected in $X$ or, alternatively, that the $\mathscr{C}$-hull of two points contain a third and be closed in $X$.

The following quite different independent set of axioms characterizing the usual convexity structure in $E^{d}$ were given in Womble's dissertation, the first three of which imply that the members of $\mathscr{C}$ are convex:

Axiom 1': $\mathscr{C}$ is closed under isometries in $X$.

Axiom 2': If $x \in C$ and $C \in \mathscr{C}$ then $\{x\}$ and $C$ can be weakly separated (in the ordinary sense) in the flat of least dimension containing $x$ and $C$.

Axiom 3': $\mathscr{C}$ is $T_{1}$

Axiom 4': $\mathscr{C}$ is finitely join-hull commutative.

Axiom 5': $\mathscr{C}$ is domain finite.

Axiom 6': For $u \in \mathscr{C}(x, y)$ and $v \in \mathscr{C}(x, z)$ then $\mathscr{C}(u, z) \cap \mathscr{C}(v, y) \neq \varnothing$

1 The restriction $x \notin \operatorname{cl} \mathscr{C}(S)$ is contrived to achieve independence. 
(see Ellis [4]).

Now we prove the assertions made previously about Axioms 1-5. We shall let $L(x, y)$ denote the line (1-flat) determined by $x$ and $y$ (if $x \neq y$ ), and $x y$ the join (ordinary convex hull) of $x$ and $y$. Recall that any two corresponding points of a direct similitude may be specified in advance.

Lemma 1. If $\mathscr{C}$ satisfies Axioms 1 and 2 then for any two distinct points $x$ and $y$,

$$
\mathscr{C}(x, y) \subset L(x, y),
$$

and $\mathscr{C}$ is $T_{1}$.

Proof. First, note the two fundamental properties of any oneto-one mapping $f: X \rightarrow X$ having the property that for each $C \in \mathscr{C}$, $f[C]$ and $f^{-1}[C]$ are members of $\mathscr{C}$ :

(i) If $S \subset X$ then $f[\mathscr{C}(S)]=\mathscr{C}(f[S])$.

(ii) If $f[S] \cup T \subset \mathscr{C}(S)$ then $f[T] \subset \mathscr{C}(S)$.

The first being routine, the second may be proved from the first by writing

$$
f[T] \subset f[\mathscr{C}(S)]=\mathscr{C}(f[S]) \subset \mathscr{C}[\mathscr{C}(S)]=\mathscr{C}(S) .
$$

In particular, (i) and (ii) hold if $f$ is any similitude. Now suppose $C=\mathscr{C}(x, y)$ and $z \in C \sim L(x, y)$. Let $f$ be a direct similitude which takes $x$ to $x, y$ to $z$, and leaves the plane of $z$ and $L(x, y)$ invariant. With $z_{-1}=y$ and $z_{0}=z$, define

$$
z_{n}=f\left(z_{n-1}\right) \quad \text { and } \quad \angle\left(z_{n-1}, x, z_{n}\right)=\theta_{n} .
$$

for $n=0,1, \cdots$. An inductive application of (ii) proves that $z_{n} \in C$ for all $n$. Note that $\theta_{n}=\theta_{0}$ for each $n$ and that, therefore, $\angle\left(z_{-1}, x, z_{n}\right)=(n+1) \theta_{0}$ for all $n$ such that $(n+1) \theta_{0} \leqq \pi$. Thus, there is an integer $n$ for which $\angle\left(z_{-1}, x, z_{n}\right)>\pi / 2$. Set $u=z_{n}$ and let $v$ be the reflection of $u$ in the perpendicular bisector of $x y$. It follows that $v \in C$ and thus $C$ contains a (perhaps degenerate) trapezoid $(x, y, v, u)$ with $e(u, v)>e(x, y)$. If $g$ is a direct similitude which maps $x$ to $u$ and $y$ to $v$, again define $u_{0}=u, v_{0}=v$, and

$$
u_{n+1}=g\left(u_{n}\right), \quad v_{n+1}=g\left(v_{n}\right)
$$

for $n=0,1, \ldots$. It follows that $u_{n} \in C$ and $x_{n} \in C$ for all $n \geqq 0$ and $e\left(u_{n}, v_{n}\right)=\lambda^{n} e\left(u_{0}, v_{0}\right)$, where $\lambda=e(u, v) / e(x, y)>1$. Thus, $C$ is unbounded. But if $B$ is the guaranteed set of Axiom 2 and $h$ is a 
similitude which maps the two guaranteed points of $B$ onto $x$ and $y$ then $h[B]$ is a member of $\mathscr{C}$ containing $\mathrm{x}$ and $\mathrm{y}$ and therefore

$$
C=\mathscr{C}(x, y) \subset h[B],
$$

contradicting the boundedness of $B$.

Lemma 1'. If $\mathscr{C}$ satisfies Axioms 1 and 2 then for any two distinct points $x$ and $y$,

$$
\mathscr{C}(x, y) \subset x y \text {. }
$$

Proof. Same proof as in Lemma 1, using a trapezoid of 0 height.

Lemma 2. If $\mathscr{C}$ satisfies Axioms 1-3, then for any finite set of points $x_{1}, \cdots, x_{k}$,

$$
\mathscr{C}\left(x_{1}, \cdots, x_{k}\right) \subset \operatorname{conv}\left(x_{1}, \cdots, x_{k}\right) .
$$

Proof. The assertion is true for $k=2$ by Lemma 1 ', so suppose it has been shown for any set of $k-1$ points, $k \geqq 3$. Let $x_{1}, \cdots, x_{k}$ be given and choose the notation so that $x_{1}$ is an extreme point of conv $\left(x_{1}, \cdots, x_{k}\right)$ and put $S=\left\{x_{2}, \cdots, x_{k}\right\}$. Thus, $x_{1} \notin$ cl conv $S$ and by the induction hypothesis $\mathscr{C}(S) \subset \operatorname{conv} S$, so $x_{1} \notin \operatorname{cl} \mathscr{C}(S)$. By Axiom 3,

$$
\begin{aligned}
\mathscr{C}\left(x_{1}, \cdots, x_{k}\right) & =\mathscr{C}\left(x_{1} \cup S\right) \subset\left(x_{1}\right)_{\mathscr{C}} \mathscr{C}(S) \subset \bigcup_{s \in \text { conv } S} \operatorname{conv}\left(x_{1}, s\right) \\
& =\operatorname{conv}\left(x_{1}, \cdots, x_{k}\right) .
\end{aligned}
$$

LeMma 3. If $\mathscr{C}$ satisfies Axioms 1-4 and $\mathscr{C}_{k}$ denotes the members of $\mathscr{C}$ lying in a flat of dimension $k$, then $\mathscr{C}_{k}$ has Helly number $k+1,1 \leqq k \leqq d$.

Proof. Using induction on the deficiency $d-k$ of the flat, suppose the assertion has been proved for any collection $\mathscr{C}_{k}$ and let $C_{1}, \cdots, C_{k+1}$ be $k+1$ members of $\mathscr{C}_{k-1}$ contained in a $(k-1)$-flat $F$, each $k$ of which have nonempty intersection. We first replace the $C_{i}$ by $\mathscr{C}$-hulls $P_{i}$ of finite point sets. For each $i$ define $x_{i} \in \bigcap_{j=1, j \neq i}^{k+1} C_{j}$ and let $P_{i}=\mathscr{C}\left(x_{1}, \cdots, x_{i-1}, x_{i+1}, \cdots, x_{k+1}\right)$. It follows that $P_{j} \subset C_{j}$ for each $j$. Each $k$ of $P_{1}, \cdots, P_{k+1}$ have nonempty intersection since $x_{i} \in P_{j}$ if $i \neq j$, and, therefore, $x_{i} \in \bigcap_{j=1, j \neq i}^{k+1} P_{j}$. Let $x \notin F$ and define the sets $C_{i}^{\prime}=\mathscr{C}\left(x \cup P_{i}\right)$ for $1 \leqq i \leqq k+1$ and $C_{k+2}^{\prime}=\mathscr{C}\left(x_{1}, \cdots, x_{k+1}\right)$. Then by Lemma 2 the sets $C_{1}^{\prime}, \cdots, C_{k+2}^{\prime}$ lie in a $k$-flat and each $k+1$ have nonempty intersection. By the induction assumption there exists $p \in \bigcap_{i=1}^{k+2} C_{i}^{\prime}$. For each $i$, one can use Axiom 3 and Lemma 2 to prove that 


$$
p \in C_{i}^{\prime} \cap C_{k+2}^{\prime} \subset F \cap x_{\mathscr{\&}} P_{i} \subset F \cap\left(\bigcup_{s \in P_{i}} x s\right)=P_{i} .
$$

The standard inductive argument may now be used to extend the property to any finite subcollection of $\mathscr{C}_{k-1}$, and hence $\mathscr{C}_{k-1}$ has Helly number $k$.

Lemma 4. If $\mathscr{C}$ satisfies Axioms 1-4, then for any finite set of points $x_{1}, \cdots, x_{k}$,

$$
\mathscr{C}\left(x_{1}, \cdots, x_{k}\right)=\operatorname{conv}\left(x_{1}, \cdots, x_{k}\right) \text {. }
$$

Proof. Consider the case $k=2$, and suppose $p \in x y \sim \mathscr{C}(x, y)$. The sets $C_{1}=\mathscr{C}(x, p), C_{2}=\mathscr{C}(p, y)$, and $C_{3}=\mathscr{C}(x, y)$ are subsets of $F=L(x, y)$ so that Lemma 3 applies, with $k=2$. Each 2 of the $C_{i}$ intersect, but $\bigcap_{i=1}^{3} C_{i}=\varnothing$ since $p \notin \mathscr{C}(x, y)$. The contradiction proves that $x y \subset \mathscr{C}(x, y)$ and establishes the result for $k=2$. Induction may then be applied to finish the proof.

The obvious result of Lemma 4 is

THEOREM 9. If $\mathscr{C}$ is a convexity structure for $X=E^{d}$ satisfying Axioms 1-4, then each member of $\mathscr{C}$ is convex.

The independence of our axioms show that at this point $\mathscr{C}$ need not contain all the convex sets of $X$. But the use of Axiom 5 together with the result of Lemma 4 provides an easy proof of the final result.

THEOREM 10. If $\mathscr{C}$ is a convexity structure for $X=E^{d}$ satisfying Axioms 1-5, then $\mathscr{C}$ is precisely the family of convex sets of $X$.

Proof. Let $C \subset X$ be convex, and consider $x \in \mathscr{C}(C)$. By Axiom 5 there exist points $x_{1}, \cdots, x_{k}$ in $C$ such that

$$
x \in \mathscr{C}\left(x_{1}, \cdots, x_{k}\right)=\operatorname{conv}\left(x_{1}, \cdots, x_{k}\right) \subset \operatorname{conv} C=C .
$$

Therefore $\mathscr{C}(C) \subset C$, implying $C=\mathscr{C}(C)$. Hence, $C \in \mathscr{C}$ and, in view of Theorem 9 , the result is proved.

It is routine to show the independence of each of the above axioms, except for Axiom 2. For, in order to deny exactly one each of the Axioms 1, 3, 4, and 5, merely take $\mathscr{C}$ to be, respectively,

(i) $\varnothing, X$, and all convex subsets of diameter $\leqq 1$;

(ii) $\varnothing, X$, and all convex subsets of dimension $\leqq k$ for some fixed $k, 2 \leqq k<d^{2}$;

${ }^{2}$ It is easy to find examples in (i) and (ii) for which there are nonconvex members of $\mathscr{C}$. This, together with Example 3, shows that no proper subsystem of Axioms 1-4 is sufficient to prove Theorem 9 . 
(iii) $\varnothing, X$, and all subsets of cardinality $\leqq d+2$; and,

(iv) all compact convex subsets of $X$.

To show the independence of Axiom 2 more effort is required. First, we observe the result: If $Q$ denotes the set of all rational points in $E^{2}$ and $f$ is a similitude of $E^{2}$, then $Q \cap f[Q]$ must be either $\varnothing$, a singleton, or $Q$ itself. This is proved by using the analytic form of a plane similitude (the plane being coordinatized by $(\xi, \eta))$ :

$$
\begin{aligned}
& \xi^{\prime}=\alpha \xi-\beta \eta+\lambda, \\
& \eta^{\prime}=\varepsilon \beta \xi+\varepsilon \alpha \eta+\mu,
\end{aligned}
$$

where $\varepsilon= \pm 1$. Then if $\left(\xi_{i}, \eta_{i}\right), i=1,2$, are points of $Q \cap f[Q]$ it may be readily shown that $\alpha$ and $\beta$ are rational and that, therefore, $\lambda$ and $\mu$ are rational (call $f$ rational in this case). Hence $f[Q]=Q$, and the result follows.

Example 3. With $X=E^{2}$, define $\mathscr{C}$ as the collection consisting of $\varnothing, X, Q$, all sets of the form $f[Q]$, where $f \in \Omega \equiv$ family of plane similitudes, and all singleton subsets of $X$.

It may be proved that $\mathscr{C}$ is a convexity structure (to show that $S=\bigcap_{i \in I} f_{i}[Q]$ is a member of $\mathscr{C}$ if $f_{i} \in \Omega$ for each $i \in I$, write $f_{j}^{-1}[S]=\bigcap_{i \in I} g_{i}[Q]$, where $g_{i}=f_{j}^{-1} f_{i}$, and thus $g_{j}[Q]=Q$; apply the above observation to show that either $f_{j}^{-1}[S]$ is a singleton or $g_{i}$ is rational for all $i$ ). Axiom 1 is valid, Axiom 2 is obviously denied, and Axiom 3 holds trivially since if $C \in \mathscr{C}$ has 2 or more points, cl $C=X$. Because of the pathological nature of the example it is remarkable that $c=3, h=3$, and $r=4$-as in classical convexity! (Thus, Axioms 4 and 5 are valid.) Leaving the other two proofs for the reader, consider the following argument for $h=3$ : Let $C_{1}, \cdots, C_{k}$ be members of $\mathscr{C}$ such that any 3 intersect (it is obvious that $h \neq 2$ by considering the $\mathscr{C}$-hulls of the pairs of three points consisting of two rationals and one irrational point). Without loss of generality, assume that $C_{i} \neq X$ and $C_{i} \neq$ singleton, for each $i$. Thus $C_{i}=f_{i}[Q], f_{i} \in \Omega$.

Case 1. $C_{1} \cap C_{i}=x$ for some $i$. Then let $j \neq i$ and consider $C_{1} \cap C_{i} \cap C_{j} \neq \varnothing$. Thus $x \in C_{j}$ and $x \in \bigcap_{i=1}^{k} C_{i}$.

Case 2. $C_{1} \cap C_{i}$ contains at least two points for each $i$. Then the set $f_{1}^{-1}\left[C_{1} \cap C_{i}\right]=Q \cap f_{1}^{-1} f_{i}[Q]$ contains at least two points so that $f_{1}^{-1}\left[C_{1} \cap C_{i}\right]=Q$ or $C_{1} \cap C_{i}=f_{1}[Q]$, for all $i$. Therefore, $f_{1}[Q] \subset \bigcap_{i=1}^{k} C_{i}$, and $\mathscr{C}$ has Helly number 3 . 


\section{REFERENCES}

1. L. Danzer, B. Grünbaum, V. Klee, Helly's theorem and its relatives, Amer. Math. Soc., Proceedings of Symposia in Pure Mathematics, 7 (1963), 101-180.

2. A. Dvoretzky, A converse of Helly's theorem on convex sets, Pacific J. Math., 5 (1955), 345-350.

3. J. Eckhoff, Der Satz von Radon in konvexen Produktstrukturen I, Monatshefte fur Mathematik, 72 (1968), 303-314.

4. J. W. Ellis, A general set-separation theorem, Duke Math. J., 19 (1952), 417-421.

5. P. C. Hammer, Kuratowski's closure theorem, Nieuw Archief voor Wiskunde, 8 (1960), 74-80.

6. — Extended topology: Carathéodory's theorem on convex hulls, Rendiconti del Circulo Mathematico de Palermo, 14 (1965), 34-42.

7. - Semispaces and the topology of convexity, Amer. Math. Soc., Proceedings of Symposia in Pure Mathematics, 7 (1963), 305-316.

8. W. Koenen, The Kuratowski closure problem in the topology of convexity, Amer. Math. Monthly, 73 (1966), 704-708.

9. F. W. Levi, On Helly's theorem and the axioms of convexity, J. Indian Math. Soc., 15 (1951), 65-76.

10. J. R. Reay, Carathéodory theorems in convex product structures, Pacific J. Math., 35 (1970), 227-230.

Received July 7, 1970. 7, 1970. Some results in this paper are from the doctoral dissertation of E. W. Womble, The University of Oklahoma (1969).

UNIVERSITY OF OKLAHOMA

AND

Presbyterian College 



\section{PACIFIC JOURNAL OF MATHEMATICS}

\section{EDITORS}

\author{
H. SAMELSON \\ Stanford University \\ Stanford, California 94305 \\ C. R. HовBY \\ University of Washington \\ Seattle, Washington 98105
}

J. DUGUndis

Department of Mathematics

University of Southern California

Los Angeles, California 90007

RICHARD ARENS

University of California

Los Angeles, California 90024

\section{ASSOCIATE EDITORS}
E. F. BeCKenbaCH
B. H. NeUmanN
F. WOLF
K. YoshidA

\section{SUPPORTING INSTITUTIONS}

\author{
UNIVERSITY OF BRITISH COLUMBIA \\ CALIFORNIA INSTITUTE OF TECHNOLOGY \\ UNIVERSITY OF CALIFORNIA \\ MONTANA STATE UNIVERSITY \\ UNIVERSITY OF NEVADA \\ NEW MEXICO STATE UNIVERSITY \\ OREGON STATE UNIVERSITY \\ UNIVERSITY OF OREGON \\ OSAKA UNIVERSITY \\ UNIVERSITY OF SOUTHERN CALIFORNIA
}

\author{
STANFORD UNIVERSITY \\ UNIVERSITY OF TOKYO \\ UNIVERSITY OF UTAH \\ WASHINGTON STATE UNIVERSITY \\ UNIVERSITY OF WASHINGTON

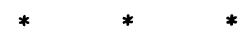 \\ AMERICAN MATHEMATICAL SOCIETY \\ CHEVRON RESEARCH CORPORATION \\ NAVAL WEAPONS CENTER
}

The Supporting Institutions listed above contribute to the cost of publication of this Journal, but they are not owners or publishers and have no responsibility for its content or policies.

Mathematical papers intended for publication in the Pacific Journal of Mathematics should be in typed form or offset-reproduced, (not dittoed), double spaced with large margins. Underline Greek letters in red, German in green, and script in blue. The first paragraph or two must be capable of being used separately as a synopsis of the entire paper. The editorial "we" must not be used in the synopsis, and items of the bibliography should not be cited there unless absolutely necessary, in which case they must be identified by author and Journal, rather than by item number. Manuscripts, in duplicate if possible, may be sent to any one of the four editors. Please classify according to the scheme of Math. Rev. Index to Vol. 39. All other communications to the editors should be addressed to the managing editor, Richard Arens, University of California, Los Angeles, California, 90024.

50 reprints are provided free for each article; additional copies may be obtained at cost in multiples of 50 .

The Pacific Journal of Mathematics is published monthly. Effective with Volume 16 the price per volume (3 numbers) is $\$ 8.00$; single issues, $\$ 3.00$. Special price for current issues to individual faculty members of supporting institutions and to individual members of the American Mathematical Society: $\$ 4.00$ per volume; single issues $\$ 1.50$. Back numbers are available.

Subscriptions, orders for back numbers, and changes of address should be sent to Pacific Journal of Mathematics, 103 Highland Boulevard, Berkeley, California, 94708.

PUBLISHED BY PACIFIC JOURNAL OF MATHEMATICS, A NON-PROFIT CORPORATION

Printed at Kokusai Bunken Insatsusha (International Academic Printing Co., Ltd.), 7-17, Fujimi 2-chome, Chiyoda-ku, Tokyo, Japan. 


\section{Pacific Journal of Mathematics}

Vol. 38, No. $2 \quad$ April, 1971

Richard Davis Anderson and Thomas Ashland Chapman, Extending

homeomorphisms to Hilbert cube manifolds .................. 281

Nguyen Huu Anh, Restriction of the principal series of $\operatorname{SL}(n, \mathbf{C})$ to some

reductive subgroups................................ 295

David W. Boyd, Indices for the Orlicz spaces . . . . . . . . . . . . 315

William Garfield Bridges, The polynomial of a non-regular digraph ...... 325

Billie Chandler Carlson, Robert K. Meany and Stuart Alan Nelson, Mixed

arithmetic and geometric means........................ 343

H. A. Çelik, Commutative associative rings and anti-flexible rings ...... 351

Hsin Chu, On the structure of almost periodic transformation groups ...... 359

David Allyn Drake, The translation groups of n-uniform translation

Hjelmslev planes ................................ 365

Michael Benton Freeman, The polynomial hull of a thin two-manifold . . . . 377

Anthony Alfred Gioia and Donald Goldsmith, Convolutions of arithmetic

functions over cohesive basic sequences .................... 391

Leslie C. Glaser, A proof of the most general polyhedral Schoenflies

conjecture possible ................................

Thomas Lee Hayden and Ted Joe Suffridge, Biholomorphic maps in Hilbert

space have a fixed point ................................ 419

Roger Alan Horn, Schlicht mappings and infinitely divisible kernels ...... 423

Norman Ray Howes, On completeness ...................... 431

Hideo Imai, Sario potentials on Riemannian spaces................ 441

A. A. Iskander, Subalgebra systems of powers of partial universal

algebras.

Barry E. Johnson, Norms of derivations of $\mathscr{L}(\mathrm{X})$.

David Clifford Kay and Eugene W. Womble, Axiomatic convexity theory and relationships between the Carathéodory, Helly, and Radon numbers

Constantine G. Lascarides, A study of certain sequence spaces of Maddox

and a generalization of a theorem of Iyer .............

C. N. Linden, On Blaschke products of restricted growth .

John S. Lowndes, Some triple integral equations ................. 515

Declan McCartan, Bicontinuous preordered topological spaces ......... 523

S. Moedomo and J. Jerry Uhl, Jr., Radon-Nikodým theorems for the Bochner and Pettis integrals ...

Calvin Cooper Moore and Joseph Albert Wolf, Totally real representations

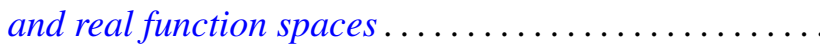

Reese Trego Prosser, A form of the moment problem for Lie groups. ... 